Recebido em: 19/05/2017

Aceito em: 21/09/2017

\section{La red de co-autores en la bibliometria Mexicana}

\author{
Co-authorship network analysis of Mexican bibliometrics
}

\author{
Rubén Urbizagástegui ALVARADo (ruben@ucrl.edu)* \\ Cristina RESTREPO ARANGO (crestrepoarango@gmail.com)** \\ * Bibliotecário Universidad de California em Riverside. \\ ** Professora em Biblioteconomia del Colégio de México.
}

Resumen: Se analiza la red de coautorías existentes en las publicaciones sobre las metrías (bibliometría, informetría, cienciometría, patentometría, etc.) en México, desde la década de los 70s hasta diciembre de 2012. Se utilizó el análisis de redes sociales a través del software Ucinet 6.0 y Netdraw. Los análisis muestran una red altamente centralizada. La densidad global de la red indica una red de baja densidad comunicacional que hace una utilización efectiva de una quinta parte de los vínculos reales sobre el total de vínculos posibles entre todos los autores de la red. El grado de centralidad identifica once autores como los componentes estructurales de la red de bibliometría mexicana. Los coeficientes del grado de cercanía indican que tanto la lejanía como la cercanía son homogéneas. En relación con el grado de intermediación se encontró que no existe una red ampliamente extendida sino la dominancia de un pequeño grupo de tres autores. La distancia geodésica identificó un único autor bien conectado a otros autores también muy bien posicionados en la red. No se identificaron cliques ni n-cliques en la red de coautoría de la bibliometría mexicana.

Palabras-clave: Bibliometría. Informetría. Cienciometría. México. Red de coautorías. Análisis de redes.

\begin{abstract}
Existing network of Mexican co-authorship in publications on metrics (bibliometrics, informetrics, scientometrics, patentometría, etc.) is analyzed, from the early 70s until December 2012. The social network analysis was done using the Ucinet 6.0 and Netdraw softwares. The analysis shows a highly centralized network. The overall network density indicates a low communication network density that makes effective use of one-fifth of the real bonds of total possible links between the authors of the network. The degree of centrality identifies eleven authors as structural components of the network of Mexican bibliometrics. The coefficient of the degree of proximity indicates that both the distance as the closeness are homogeneous. Regarding the degree of intermediation is found that there is a widespread network but the dominance of a small group of three authors. The geodesic distance identified a single author well connected to other authors also very well positioned in the network. No cliques nor ncliques in the network of co-authorship of Mexican bibliometrics were identified.
\end{abstract}

Keywords: Bibliometrics. Informetrics. Scientometrics. Mexico. Authorship Network. Network Analysis.

\footnotetext{
v. 23, n. $51,2018$. p. 74-94
}

ISSN 1518-2924
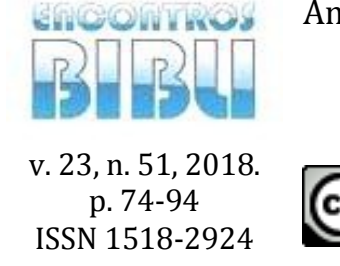
Esta obra está licenciada sob uma Licença Creative Commons 


\section{INTRODUCCIÓN}

El objetivo de este artículo es analizar la red de coautorías existentes en las publicaciones sobre las metrías (bibliometría, informetría, cienciometría, patentometría, etc.) en México. El periodo escogido se extiende desde los primeros trabajos publicados en la década de los 70s hasta diciembre de 2012, un periodo extenso como para esperar que la literatura publicada se acumule, sedimente y muestre algún tipo de colaboración de sus productores. Recientemente Urbizagástegui \& Restrepo (2014) mostraron que la colaboración de los autores en la bibliometría mexicana está en aumento y que la literatura crece a una tasa anual del $8.2 \%$ duplicando su tamaño cada 9 años. Por lo tanto, es una ocasión propicia para analizar las colaboraciones en la producción de literatura científica en el país.

Se llama colaboración al hecho de que dos o más autores publican juntos un artículo académico. Esta palabra "colaboración" tiene su origen en el latín Collaborāre que significa trabajar con otra u otras personas en la realización de una obra y se define como la "acción y efecto de colaborar" (Diccionario de la lengua española, 2001). Los científicos colaboran cuando comparten datos, equipos e ideas en un proyecto de investigación o en prácticas experimentales y análisis de investigaciones, cuyos resultados pueden ser publicados en la forma de artículos u otras publicaciones (Katz \& Martin, 1997). Una vez publicados esos artículos tendrán autorías, por lo tanto las autorías se convierten en unidades de análisis de una publicación académica. Por esa razón, los autores que contribuyen con documentos publicados en un determinado campo científico se constituyen en una población que cuando se estudian sus características surgen patrones de colaboración en la forma de redes de autores colaboradores. Esas redes de autores consisten en un conjunto de actores que establecen determinadas relaciones en función de un objetivo común. Por lo tanto, para entender adecuadamente una red de actores (autores) se hace necesario realizar una descripción de la estructura de sus relaciones subyacentes y no visibles a simple vista. El estudio de estas relaciones subyacentes de actores se efectúa a partir de la teoría de grafos y la teoría de matrices que permiten representar y describir una red de manera sistemática posibilitando clarificar determinados comportamientos de los actores (Hanneman \& Riddle, 2005).

Una red de colaboración científica de autores supone acciones de cooperación que generen un proyecto de investigación y compartir ideas y objetivos comunes. Una de las formas de garantizar la continuidad y reproducción de una comunidad científica se produce por medio de la formación de grupos especializados que repercuten tanto en el incremento de la producción científica como en su consolidación. Según Fleck (1979), los conocimientos se construyen socialmente mediante los llamados "colectivos de pensamiento", es decir, agrupaciones de individuos que intercambian ideas o mantienen relaciones de intercambio intelectual. Estos colectivos de pensamiento posibilitan la expresión de un "estilo de pensamiento", como un sistema común de creencias que se consideran como evidentes al aplicar un mismo método como forma de cognición, en otras palabras, los especialistas son siempre "socializados" en el estilo de pensamiento de su colectivo. Según Price \& Beaver (1966), la colaboración se produce en el ámbito de los "colegios invisibles", comunidades informales de investigadores que se comunican entre sí, intercambian informaciones y publican formalmente los resultados de sus investigaciones. Por lo tanto, la estabilidad de una línea de investigación en torno a un grupo de investigadores, es indicio de la existencia de una especie de "colegio invisible", un colectivo de autores que desarrolla sus actividades bajo la influencia y dirección de un líder especializado y socializado en el "estilo de pensamiento". Este líder es reconocido en el campo y tiene una gran productividad académica tanto que en la opinión de Crane (1972) los indicadores más importantes de la organización social en 
una área de investigación serían la discusión informal de las investigaciones en proceso, la publicación en colaboración, las relaciones entre los investigadores y la influencia de los colegas en la definición de los problemas y técnicas de investigación utilizadas en el campo. En torno de este colegio invisible se establece una red de comunicación tanto formal como informal, en la que los integrantes forman círculos sociales de colaboración directa o indirecta, de esa manera se genera un estilo de pensamiento colectivo. Los colegios invisibles pueden manifestar distintas jerarquías entre sus miembros, según las trayectorias individuales y los desempeños profesionales. Las posiciones centrales de un colegio están ocupadas por los autores de mayor producción y visibilidad, ellos actúan como principales receptores y difusores de información (Iñiguez, et al., 2006). En ese sentido, la "colaboración científica puede ser un emprendimiento cooperativo que envuelve metas comunes, esfuerzos coordenados y resultados o productos con responsabilidad y méritos compartidos" (Balancieri, et al., 2005, p.64).

Las relaciones entre los miembros de un colegio invisible son registradas por medio de la co-autoría de documentos publicados. Dos o más científicos son considerados conectados si publicaron un documento de forma conjunta. Así en una red de colaboración científica los nudos son los científicos y dos de ellos están conectados si colaboraron en la publicación de un documento científico (Barabási, et al., 2002), una red de colaboración científica es así el reflejo de las relaciones e interacciones existentes entre los investigadores. El análisis de esas redes permite el estudio de las comunidades especializadas y de los colegios invisibles. Para el análisis de este tipo de estudios se utiliza la técnica del análisis de redes sociales, pues, en la era de la sociedad de la información las funciones y los procesos dominantes cada vez más se organizan en redes y estas redes serían como estructuras abiertas capaces de expandirse ilimitadamente, integrando nuevos nudos cuando consiguen conciliar objetivos en la red. De esa manera una estructura social organizada en red sería un sistema dinámico, abierto, susceptible de innovaciones y sin amenazas a su equilibrio (Castells, 2009). Sobre este aspecto Ziman (1979) presenta a la ciencia como una actividad eminentemente social afirmando que la comunicación de las ideas de los científicos se establecen a través de relaciones sociales, y que por esa razón para entender la naturaleza de la ciencia es necesario observar la manera como los científicos se comportan, como se relacionan y como transmiten las informaciones o se comunican entre sí. A Price (1963) le debemos la idea de que el científico solitario encerrado en su laboratorio está en proceso de desaparición, que la ciencia es hecha por grupos que forman colegios invisibles para el constante intercambio de informaciones con relación a sus investigaciones aunque estos científicos estén localizados en instituciones y países diferentes y lejanos, es decir, en constante inter-relaciones. Para Zuccala (2006) el colegio invisible es la estructura organizativa de un conjunto de actores sociales que intercambian información formal e informal ajustándose a las reglas de una disciplina científica y a los problemas de investigación. También el concepto de relaciones es central a la sociología reflexiva propuesta por Bourdieu \& Wacquant (2005, p. 150) quien afirma que "en términos analíticos, un campo puede ser definido como una red o una configuración de relaciones objetivas entre posiciones. Estas posiciones están objetivamente definidas, en su existencia y en las determinaciones que imponen sobre sus ocupantes, agentes o instituciones, por su situación presente y potencial (situs) en la estructura de distribución de especies del poder (o capital) cuya posesión ordena el acceso a ventajas específicas que están en juego en el campo, así como por su relación objetiva con otras posiciones (dominación, subordinación, homología, etcétera)". Para construir ese sistema de relaciones entrelazadas, dos cosas son importantes: en primer 
lugar, el sistema tiene que ser completo, esto es, toda la población de elementos relevantes tiene que ser llevada en consideración; en segundo lugar, los elementos tienen que estar ligados unos a los otros por medio de relaciones internas de tal modo que no puedan ser definidos independientemente unos de los otros, de manera que estén mutua y conceptualmente implicados unos con los otros. Pero estas redes sociales están ligadas a un capital social definido como "la suma de los recursos reales o potenciales que están vinculados a la posesión de una red duradera de relaciones más o menos institucionalizas de conocimiento o reconocimiento mutuo" (Bourdieu, 1985, p. 248) y que pueden ser convertibles en diferentes formas de capital. Por lo tanto, las redes sociales se constituyen en espacios donde circulan bienes y servicios de diferentes tipos, espacios donde se establecen lazos sociales particulares que solo pueden ser comprendidos a partir de la interacción de quienes participan de estos vínculos. Sin embargo, "la red social no es más que un modo en que se estructura la interacción como el resultado de las estrategias de los agentes, que son a su vez el resultado de los habitus de éstos. Esta estructura emergente de la red no se confunde con la estructura del espacio social ni con la de un campo en particular. Simplemente, es otra estructura reticular que se sitúa en el nivel de la interacción, forma parte del conjunto de condiciones de todo tipo dentro de las cuales los agentes van creando y recreando sus cursos de acción" (Baranger, 2000, p. 47). De lo expresado en los párrafos anteriores se pueden plantear las preguntas de:

a) ¿Existe una red de colaboración en la forma de coautorías en la bibliometría mexicana?

b) ¿Es posible identificar las figuras centrales en esa red de coautorías?

c) ¿Qué autores son las figuras centrales en esa red de coautorías?

Para responder esas preguntas de investigación, este artículo está organizado de la siguiente manera: en la primera parte se hace una introducción al problema de investigación y se formulan las preguntas. En la segunda parte se revisa la literatura pertinente. En la tercera parte se describe la metodología, es decir, las unidades de análisis, la forma de recolección de los datos y la forma de medición de los mismos. En la cuarta parte se describen los resultados obtenidos y las conclusiones. Finalmente se presenta la literatura revisada en la redacción de esta investigación.

\section{REVISIÓN DE LA LITERATURA}

Las redes pueden ser consideradas estructuras capaces de expandirse indefinidamente cuando sus actores se comunican, integrando nuevos nodos y fomentando la colaboración. Consisten de un actor que puede ser una persona, un grupo de personas, organizaciones, conceptos, enlaces $\mathrm{u}$ otras colectividades. Los lazos establecen las conexiones entre pares de actores (Newman, 2000; Borgatti y Foster, 2003). Entre los indicadores de colaboración científica se encuentra la coautoría que ha sido utilizada para evaluar la colaboración entre instituciones, países y/o científicos (Leta \& Cruz, 2003), por medio del análisis de las redes sociales. Este tipo de análisis de redes sociales por medio de la bibliometría han sido aplicadas a una serie de campos del conocimiento tales como la ciencia de la información en el Brasil (Parreiras, et al., 2006), sus resultados indican concentración en las publicaciones en la forma de artículos académicos de autores únicos y transitantes. También Silva, et al. (2006) analizaron la producción en coautoría de los artículos científicos de los programas de post-graduación en ciencia de la información en el Brasil. Los artículos fueron recolectados de revistas y congresos del área registrados en la base de datos RedeCI. La 
vinculación de los profesores y sus líneas de investigación fueron levantadas a partir del sitio web de la ANCIB (Associação Nacional de Pesquisa e Pós-Graduação em Ciencia da Informação). Fueron seleccionados 116 profesores de 9 instituciones, 54 profesores que presentaron trabajos en coautoría en los artículos y ponencias presentadas en congresos. A estos 54 profesores les fue aplicada la metodología de análisis de redes sociales para la identificación de grupos (clústeres) de autores y el cálculo de las medidas de centralidad. El análisis de la red indica que los profesores poseen una baja densidad, con apenas 3,3\% de los lazos posibles presentes. Esa baja conexión se refleja en la fragmentación en 15 componentes, con cinco de ellos compuestos por más de tres profesores, indicando una baja cooperación entre ellos.

Luego serían Pinto, et al. (2007) quienes realizaron un análisis de las redes sociales en la coautoría de 635 documentos sobre bibliometría y cienciometría publicada entre 1975 y 2005, recuperadas del Science Citation Index, Social Science Citation Index y Arts \& Humanities Index. En bibliometría encontraron un punto central comandado por Thellwall, Kostoff y una relación más simple entre Borkenhagem y Brahler, y entre Guerrero y Faba-Perez. En cientometría observaron una cooperación destacada entre Padhgi y Garg, y entre Welljamsdorof y Garfield. Distinguieron una relación mixta entre Holden, Rosenberg y Barker. En lo que se refiere al consumo de información el análisis de los documentos citados corresponde a la formación teórica y práctica de los estudios métricos, con documentos citados desde 1926 (Lotka) hasta los más contemporáneos como Cronin y Borgman. Los autores más representativos muestran centralidad en Narin, Cronin, McCain, Bordons y Seglen. Observaron también que Lotka, Bradford, Price, Narin, Garfield, White, Cronin, Van Raan y Glanzel son los más citados.

Graeml, Macada y Rosson (2010), a partir de las informaciones bibliométricas de los artículos publicados en los anales del EnANPAD en el período 1997 a 2006, en el área de administración de la información (ADI), estudiaron las redes de coautoría y cocitación buscando la estructura social y la estructura intelectual del área, así como las posibles influencias de la coautoría sobre la co-citación. Bufrem, et. al. (2011) buscaron describir el estado actual y la configuración del asunto "redes sociales" en la producción académica brasileña en el campo de la ciencia de la información. Los autores identificaron y analizaron el objeto de estudio con una aproximación bibliométrica y utilizaron la literatura publicada de 1997 a 2010 indizada en la base de datos BRAPCI. El trabajo de Nascimento (2011) tenía como objetivo conocer cómo se configuran la autoría y la colaboración científica entre los autores/investigadores que publican sus trabajos en 6 revistas brasileñas del área de ciencia de la información en el período de 2000 a 2010. Verificó que las relaciones de coautoría por medio del análisis de redes sociales se muestran poco densas y repletas de lazos débiles. Concluye que los autores no exploran muchas de las posibilidades de colaboración inclusive dentro de su propia institución.

En los Estados Unidos, Zoss (2012) utilizó el análisis de coautorías para explorar las relaciones y los patrones de publicación de cinco investigadores americanos y que publicaron solo en inglés sobre bibliometría: Christine Borgman, Blaise Cronin, Eugene Garfield, Katherine McCain y Howard White. Los metadatos de las publicaciones de estos cinco investigadores fueron recogidos del Web of Science desde 1955 hasta 2011. Los datos dan una idea de cómo el campo ha evolucionado con el tiempo y la cantidad de trabajos que han sido hechos por estos cinco investigadores que son apoyados por otros estudiosos que hacen un trabajo similar.

En China, Yan \& Ding (2009) estudiaron la red de coautorías tomando datos de 16 revistas del campo de la biblioteconomía y ciencias de la información de 1988 a 
2007, recopiladas del WoS, con un total de 10,344 artículos producidos por 10,579 autores. Construyeron una red de coautoría y calcularon cuatro medidas de centralidad (cercanía, intermediación, grado y PageRank) para los autores en esta red. Encontraron que las cuatro medidas de centralidad se correlacionaron significativamente con el número de citas. Discuten la utilidad de las medidas de centralidad en el ranking de autor, y sugieren que las medidas de centralidad pueden ser indicadores útiles para el análisis de impacto de las publicaciones de los coautores. Estos autores señalan que la red de coautorías ha sido intensamente estudiada, pero la mayor parte de estas investigaciones se centran en las propiedades de nivel macro de la red, pero se ha prestado poca atención al nivel micro que proporciona mayor información sobre las limitaciones y oportunidades diferenciales que enfrentan los actores individuales y que dan forma a su comportamiento social en la estratificación social y la desigual posición de los autores en el campo.

En España, Delgado López-Cózar, et al. (2006) exploraron el análisis de redes sociales para detectar la existencia de escuelas científicas y redes académicas en las tesis doctorales sobre bibliometría leídas en universidades españolas entre 1976 y 2002. Establecieron la evolución, el perfil temático, el perfil institucional y geográfico de las tesis bibliométricas, así como identificaron a los protagonistas en la gestación de los focos de investigación bibliométrica y establecieron las posibles genealogías académicas. Los indicadores de redes sociales confirmaron el papel central de ciertos pioneros de la bibliometría en España, y revelaron cómo se difundieron estas ideas entre los diversos actores. En un determinado periodo estos actores ocuparon posiciones relevantes, pero en una última etapa fueron desplazados por el surgimiento de otras escuelas y de otras figuras más polivalentes. También el campo de la bibliotecología y la documentación española fue explotada por Vargas-Quesada, et al. (2010) analizaron la estructura de la colaboración científica española con datos de la base de datos Scopus desde 1999 hasta 2007. Para ello combinaron procedimientos de análisis de redes sociales con métodos bibliométricos como la co-ocurrencia de autores y el análisis de frecuencia de términos obtenidos de los títulos y palabras clave de los artículos. Identificaron 21 componentes conformados por 28 subredes que revelan la estructura de la red de colaboración científica. En la red observaron una colaboración centralizada en unos pocos componentes, originada en el sector investigador y que tiene como frentes de colaboración la recuperación de información y gestión de la información.

Este tipo de análisis no ha estado ausente en México; por ejemplo, Aguado-López, et al. (2009) realizaron un análisis de las redes de colaboración de coautorías utilizando artículos publicados entre 2006 y 2007 por cinco revistas editadas por la Universidad Autónoma del Estado de México (UAEM), por medio del Sistema de Información Científica REDALYC. Estudiaron la colaboración científica entre países, instituciones y autores, considerando el origen de las adscripciones desde un contexto general (país) hasta la cooperación entre autores. Collazo-Reyes, Luna Morales \& Luna Morales (2012) analizaron las redes de coautoría con miras a identificar cambios en la conformación de las estructuras de relaciones de colaboración, acumulado por los investigadores en términos de la conformación de relaciones productivas de colaboración duraderas, el tipo y el volumen de trabajos publicados por el Departamento de Fisiología, Biofísica y Neurociencias. Para recopilar los datos utilizaron la plataforma Web of Science de 1961 hasta 2010. Sin embargo, hasta donde es del conocimiento de los autores de este artículo, las exploraciones del análisis de redes sociales no han tomado como campo de investigación y exploración a los documentos producidos en el área de la bibliometría mexicana. 


\section{MATERIAL Y MÉTODOS}

Para identificar a los autores productores de documentos sobre este asunto se hizo una búsqueda en los títulos, palabras clave y resúmenes con múltiples combinaciones booleanas usando los términos listados en el Anexo A en las bases de datos bibliográficas que aparecen mencionadas en el Anexo B. Las referencias encontradas fueron exportadas a una base de datos bibliográfica en el software de administración de bibliografías EndNote X5. Cada uno de los trabajos encontrados se leyó, con el fin de verificar si el tema tratado tenía relación con las palabras clave usadas en esos documentos. De esas publicaciones también se revisaron las referencias bibliográficas para detectar otros trabajos sobre bibliometría, cienciometría o informetría, etc. no recuperados por medio de las bases de datos que se utilizaron en la búsqueda. Para identificar a los autores mexicanos se hizo un seguimiento de la afiliación institucional mencionada en cada documento recuperado. Muchas veces fue necesario buscar currículos académicos en Internet para identificar y asegurarse que los autores eran mexicanos o extranjeros afiliados a instituciones mexicanas, así como autores mexicanos que publicaron en el extranjero y autores extranjeros que publicaron en México. En algunas ocasiones fue necesario contactarlos por medio del correo electrónico. De esa manera fueron recopilados artículos publicados en revistas académicas, cartas al editor, artículos publicados en periódicos, ponencias presentadas en eventos nacionales y/o extranjeros y capítulos de libros que trataran sobre la aplicación de las técnicas relacionadas con la bibliometría, la cienciometría, la informetría, etc. producidos por autores mexicanos y extranjeros en el país y por mexicanos en el extranjero. Se excluyeron libros, tesis y literatura gris, porque estos tipos de documentos no son indizados en las bases de datos bibliográficas consultadas. Este trabajo cubre el periodo que va desde los primeros trabajos publicados en la década de 70s hasta diciembre del 2012.

Con los datos recolectados en EndNote se creó una matriz de co-autoría en Excel que con la información estructural pertinente fueron convertidos a una matriz de adyacencia para poder aplicar las métricas del análisis de redes con UCINET 6, el software usado para el análisis de las redes de coautoría. Una vez obtenidas las matrices de adyacencia, se estimó la densidad, centralidad (grado, cercanía, e intermediación) con UCINET 6. Las tablas resultantes fueron analizadas y un reporte con la interpretación de esos resultados fue creado para cada autor. Para la visualización de la red se utilizó el software NetDraw. Para el análisis de la red de coautoría de la bibliometría mexicana se consideraron las relaciones entre los autores como nodirecionadas. Como los autores publican en colaboración, se considera la existencia de reciprocidad en las respectivas publicaciones. Por lo tanto, se adoptaron las siguientes medidas de análisis de las informaciones recolectadas: la densidad de la red, que permite la visualización de la intensidad de la cooperación en la red analizada, pues, muestra la potencialidad de la red en términos de flujo de informaciones; la distancia, que indica el esfuerzo que hace un autor para alcanzar a otro autor en la red y mide la distancia media de los autores para llegar a todos los demás autores; el grado de centralidad indica el número de conexiones que salen o parten desde un autor, es decir, la cantidad de conexiones que éste tiene con sus pares; el grado de intermediación, que mide el grado en que un autor actúa como puente entre otros autores de la red, ya que analizando a todos los pares de autores se identifica al autor que se localiza en el camino más corto entre otros dos autores en la red; el grado de cercanía, que se usa para conocer la capacidad que tiene un autor de colaborar con los demás autores 
definiéndose como la capacidad que en promedio tiene un autor de estar más cerca a los otros autores en la red, es decir, la distancia promedio existente entre un autor y los otros autores de la red, finalmente la distancia geodésica (Eigenvector), que es una forma de identificar a los autores más centrales, es decir, aquellos con menos lejanía que los otros. Es una medida de la popularidad o centralidad de los autores conocidos.

\section{RESULTADOS}

Se encontraron 459 documentos producidos y publicados hasta diciembre del 2012. El 37\% de esos documentos (171) fueron producidos de forma individual, es decir, sin colaboración; por lo tanto 288 (63\%) documentos fueron producidos en colaboración por dos $(25 \%)$, tres $(21 \%)$, cuatro $(9 \%)$, cinco $(6 \%)$, etc. autores. Apenas $17 \%$ de los documentos (77 publicaciones) fueron producidos por cuatro o más autores colaboradores. La Figura 1 muestra el gráfico de barras de esta forma de colaboración en la producción de documentos sobre la bibliometría en México. La media de colaboración fue de 5 autores por documento, con un error patrón de 0.996, una desviación estándar de 2.8 y una varianza de 7.9 documentos. Sin embargo, debe entenderse que 10 autores colaboraron en la producción de dos documentos y 9 autores en la publicación de un documento, 7 autores en la producción de tres documentos y así sucesivamente.

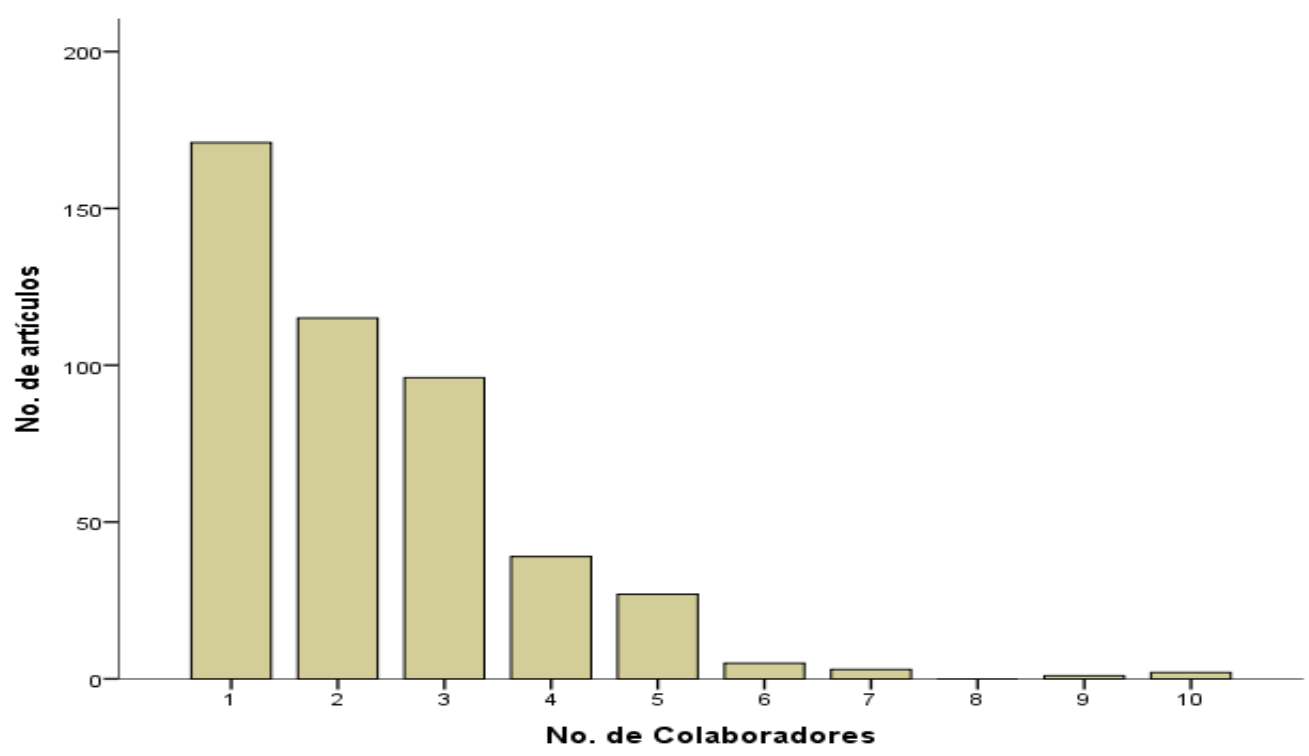

Figura 1: Número de colaboradores según el número de documentos producidos Fuente: Datos de los autores.

La Tabla 1, presenta los autores que produjeron 5 o más documentos en el periodo de estudio así como la cantidad de documentos producidos en colaboración. De los 91 documentos producidos por la autora más productiva (Jane Russell Barnard), $67 \%$ fueron producidos en colaboración. De los 42 documentos producidos por Judith Licea de Arenas, 91\% lo fueron en colaboración, y así sucesivamente la mayoría de los autores más productivos publicaron en colaboración en más del $50 \%$ de los documentos respectivos y en algunos casos hasta en el 100\% de ellos como es el caso de los que participaron en la producción de 5 documentos cada uno. La única excepción es 
la producción de Salvador Gorbea Portal que más parece un productor individual pues solo el 18\% de su producción total lo hizo en colaboración.

Tabla 1: Autores productores y producción en colaboración

\begin{tabular}{|c|c|c|}
\hline Autores & $\begin{array}{c}\text { Producción } \\
\text { total }\end{array}$ & $\begin{array}{l}\text { Producción } \\
\text { en colaboración }\end{array}$ \\
\hline Russell Barnard, Jane Margaret & 91 & 61 \\
\hline Licea de Arenas, Judith & 42 & 38 \\
\hline Gorbea Portal, Salvador & 27 & 5 \\
\hline Macías-Chapula, César Augusto & 23 & 10 \\
\hline Rio, José Antonio del & 19 & 19 \\
\hline Valles Valenzuela, Javier & 19 & 19 \\
\hline Collazo Reyes, Francisco & 18 & 15 \\
\hline Arenas Vargas, Miguel & 16 & 16 \\
\hline Narváez Berthelemot, Nora & 16 & 15 \\
\hline Luna Morales, María Elena & 16 & 14 \\
\hline Michan Aguirre, Layla & 14 & 9 \\
\hline Aguado López, Eduardo & 11 & 11 \\
\hline Urbizagástegui Alvarado, Rubén & 11 & 7 \\
\hline Ainsworth, Shirley & 10 & 10 \\
\hline Fuentes Navarro, Raúl & 10 & 9 \\
\hline Cortés, Héctor Daniel & 10 & 10 \\
\hline Rogel Salazar, Rosario & 10 & 10 \\
\hline López Ornelas, Maricela & 8 & 4 \\
\hline García, Esther Ofelia & 8 & 8 \\
\hline Guzmán Sánchez, María Victoria & 7 & 7 \\
\hline Rodea Castro, I. P. & 7 & 7 \\
\hline Pérez Angón, Miguel Ángel & 7 & 6 \\
\hline Restrepo Arango, Cristina & 7 & 5 \\
\hline Alonso Gamboa, José Octavio & 6 & 5 \\
\hline Galina Russell, Isabel & 6 & 6 \\
\hline Kostoff, Ronald N. & 6 & 6 \\
\hline Reyna Espinosa, Felipe Rafael & 5 & 3 \\
\hline Ramírez Romero, Ana María & 5 & 5 \\
\hline Sotolongo Aguilar, Gilberto & 5 & 5 \\
\hline Musi-Lechuga, Bertha & 5 & 5 \\
\hline Ramírez Godoy, Maria Esther & 5 & 5 \\
\hline Olivas-Ávila, José Alonso & 5 & 5 \\
\hline Mendoza Guerrero, J. A. & 5 & 5 \\
\hline Gutiérrez Carrasco, A. & 5 & 5 \\
\hline
\end{tabular}

Fuente: Datos de los autores.

El análisis de las redes sociales se basa en las relaciones entre los actores que se pueden visualizar, por medio de un gráfico de conexiones, para lograr esa visualización los grupos se forman sobre la base de patrones de relaciones establecidas entre los actores. Una fuerte relación directa entre los actores aumenta la probabilidad que puedan ser agrupados conjuntamente. En el caso de los autores, cuanto más documentos científicos producen en colaboración las relaciones se convierten en más cohesivas, formando un grupo identificable de colaboradores. La red de la bibliometría mexicana está compuesta de 194 nodos (autores) y 288 ligaciones (links). La distancia geodésica media de esta red es de $1.8( \pm 0.8)$ lo que significa que los autores en media tienen una distancia de 2 autores entre ellos. El coeficiente de clusterización general fue 
igual a 0.733 , indicando que si dos autores (digamos A y B) han colaborado con $\mathrm{C}$ en la coautoría de un documento por separado, existe $73 \%$ de posibilidades de que A y B autores colaboren en un futuro cercano.

La Figura 2 muestra la red de co-autorías de la bibliometría mexicana. Esta red se construyó tomando en consideración solamente a aquellos autores con 3 o más documentos producidos en colaboración. Los nudos fueron posicionados con el auxilio del algoritmo respectivo disponible en Netdraw y fueron reposicionados para evitar superposiciones innecesarias. El tamaño de los nodos es proporcional al número de artículos de los autores y el grosor de los vínculos es proporcional al número de coautorías. Una línea más gruesa entre dos autores indica que estos han publicado más documentos en coautoría. No se muestran los nodos aislados, sino sólo los nombres de los autores con más de tres documentos publicados en coautoría.

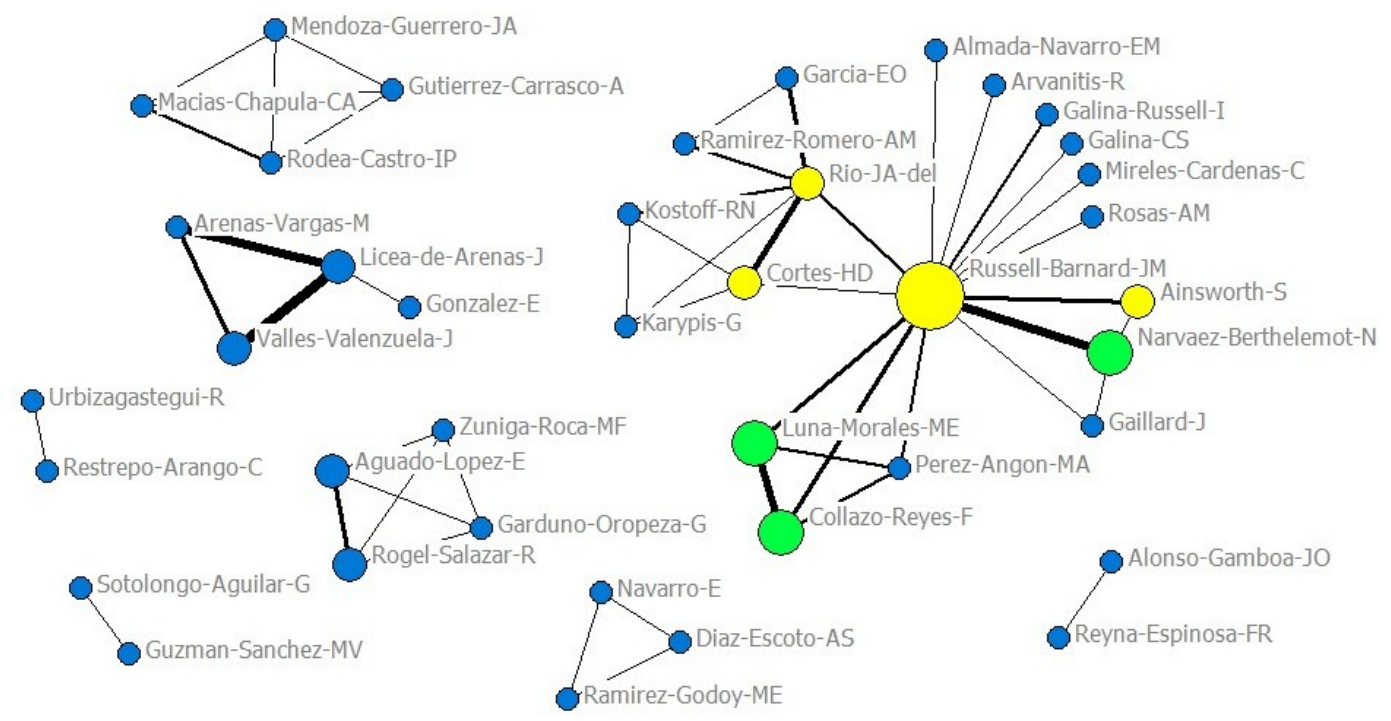

Figura 2: Red de co-autoría en la bibliometría mexicana

Fuente: Datos de los autores.

En general, la red se compone de 8 grupos bien diferenciados. Un grupo central con la posición dominante de Russell-Barnard-Jane-M, que incluye en su red a otros 18 autores colaboradores, seguido de un segundo grupo liderado por Licea-de-ArenasJudith con otros tres autores en su grupo, un tercer grupo liderado por Aguado-LópezEduardo, otra liderado por Macías-Chapula, CA y una menor liderada por RamírezGodoy-RM. Luego aparecen tres grupos de dos autores colaboradores marginales y aislados del resto de los miembros de la red. El grupo dominante está altamente centralizado alrededor de una única autora, pero paradójicamente esa centralización es una muestra de su lado débil, ya que la eventual remoción del nudo central podría producir una rápida fragmentación de este grupo en pequeños nudos aislados, por lo tanto ese nodo central también es un posible punto de ruptura. Como esta autora ejerce la docencia y como tal tiene dirigidos, estos dirigidos han sido sus eventuales colaboradores; sin embargo, el eventual retiro de esta autora de la docencia podría generar la dispersión de esta red, en dos o tres pequeños grupos de investigadores separados. Por ejemplo, debido a la relación entre Luna-Morales-ME y Collazo-Reyes-F, 
estos podrían asegurar la continuidad de este grupo de investigación. Otro grupo que podría sobrevivir e independizarse podría ser el liderado por Rio-JA-del y Cortés-HD.

\subsection{Densidad de la red}

El análisis de la densidad tiene como objetivo mostrar el valor (en porcentajes) de la conectividad de la red, es decir, si esta tiene alta o baja densidad. La densidad muestra la potencialidad de la red en términos de flujo de informaciones. Cuanto más alto el valor de la densidad más intensa será el intercambio de informaciones en la red y viceversa, cuanto más baja menor será el intercambio de informaciones en la red. La densidad de la red es definida como el porcentaje estimado de la división entre el número de lazos observados y el número máximo de conexiones posibles en la red. Si todo agente estuviese conectado directamente con cualquier otro agente se tendría una red completa y compacta. La densidad de una red es alta si muchos autores están conectados entre sí. En una red de colaboración científica, la densidad mide la proporción de colaboraciones distintas existentes sobre el total de colaboraciones distintas posibles. Cuantifica una estimación de la intensidad de la colaboración en el conjunto de la red. Cuanto más conexiones existan en la red, más productiva será en su conjunto y cuanto mayor las interrelaciones mayor la unión entre los autores. Un nivel medio de conexiones muestra la existencia de un flujo medio de informaciones entre los autores presentes en la red. La densidad alude al volumen de interconexiones entre los miembros de una red.

Para la red de coautoría de la BM se encontró que la densidad global de la red (proporción entre los vínculos reales y los potenciales) fue del $4.6 \%$ con un desvío patrón de 0.445 . Esta medida indica que estamos en presencia de una red con una densidad comunicativa bastante baja (media sería alrededor del $50 \%$ y alta, mayor al $80 \%)$ que hace una utilización efectiva de menos de una décima parte de los vínculos reales sobre el total de vínculos posibles entre todos los autores de la red. En otras palabras, solo se están utilizando el 5\% de las relaciones posibles y se están perdiendo el $95 \%$ de ellas.

\subsection{Grado de centralidad}

El grado de centralidad de un nodo se define como el número total de aristas que están adyacentes a este nodo. El grado de centralidad mide cuántos vínculos conectan a los autores con sus vecinos inmediatos en la red, es decir, que "el grado de centralidad es igual al número de conexiones que un actor (un nodo) tiene con otros actores (autores)" (Sadoughi, et al., 2016). Los autores más centrales son aquellos que tienen más conexiones con los otros autores en la red y por lo tanto son los más activos en el sentido de colaboración.

En la Tabla 2, la autora Russell-Barnard-JM tiene el mayor grado de centralidad (102) y un grado de entrada normalizado del 5.8\%, por lo tanto, juega un papel central en la BM, es la autora que más ha colaborado con otros autores, la que tiene el mayor número de conexiones; su grado de centralidad duplica en volumen al siguiente más cercano; esto significa que esta autora es la que recibe más solicitaciones de colaboración por parte de otros autores; luego vendrán el autor Rio-JA-del (53, 3\%), Licea-de-Arenas-J $(43,2.5 \%)$ y así sucesivamente. En esta tabla se muestra solo a un grupo parcial de autores, aquellos que alcanzaron un grado de centralidad igual y mayor a 15. 
Tabla 2: Grado de centralidad

\begin{tabular}{lrr} 
Autores & Grado & nGrado \\
\hline Russell-Barnard-JM & 102 & 0.058 \\
Rio-JA-del & 53 & 0.030 \\
Licea-de-Arenas-J & 43 & 0.025 \\
Cortés-HD & 36 & 0.021 \\
Narvaez-Berthelemot-N & 32 & 0.018 \\
Collazo-Reyes-F & 29 & 0.017 \\
Luna-Morales-ME & 28 & 0.016 \\
Kostoff-RN & 27 & 0.015 \\
Valles-Valenzuela-J & 27 & 0.015 \\
Arenas-Vargas-M & 26 & 0.015 \\
Karypis-G & 21 & 0.012 \\
Ainsworth-S & 18 & 0.010 \\
Leydesdorff-L & 18 & 0.010 \\
Malpohl-G & 18 & 0.010 \\
Smith-A & 18 & 0.010 \\
Smith-C & 18 & 0.010 \\
Tshiteya-R & 18 & 0.010 \\
Wagner-C & 18 & 0.010 \\
Aguado-López-E & 17 & 0.010 \\
Rogel-Salazar-R & 17 & 0.010 \\
Macías-Chapula-CA & 16 & 0.009 \\
García-EO & 15 & 0.009 \\
Pérez-Angón-MA & 15 & 0.009
\end{tabular}

Fuente: Datos de los autores.

\subsection{Grado de Intermediación}

La intermediación se basa en el número de rutas más cortas que atraviesa un actor para conectarse con otros actores. Los actores propietarios de una alta intermediación ejecutan la tarea de vincular varios grupos, como "intermediarios". El grado de intermediación mide el grado en que un autor actúa como puente entre otros autores de la red. Analizando todos los pares de autores se identifica al autor que se localiza en el camino más corto entre otros dos autores. Cuanto más se coloca en la ruta más corta a otros autores mayor será la intermediación y el autor será visto más como un "intermediario"; altos valores de intermediación significan que otros autores tienen que pasar a través de este autor para conectarse con otros autores. Por ejemplo, un autor que tenga un grado de intermediación igual o mayor a 100 sería un monopolizador de informaciones, por lo tanto tendrá la habilidad de reservar o cambiar la información pasada a través de éste. Los autores con un mayor grado de intermediación están en una situación de poder, ya que controlan mayores flujos de comunicación entre los autores. Un autor que tenga una intermediación estandarizada igual a cero nunca se colocará en el camino más corto entre dos autores colaboradores. Los autores que se colocan en los caminos más cortos entre pares de autores son vistos como los más centrales en la red de colaboración, ya que más autores dependen de éste para hacer conexiones con el resto de autores de la red.

La Tabla 3 muestra los valores de las medidas de intermediación que se obtuvieron para los autores colaboradores de la BM. Se identifica a un autor (RussellBarnard-JM) con un alto grado de intermediación, más del doble del autor siguiente (Rio-JA-del). Luego vienen un pequeño grupo de tres investigadores (NarvaezBerthelemot-N, Michan-Aguirre-L y Ramírez-Godoy-ME) con un mediano grado de 
intermediación. Estos datos muestran que no existe una red ampliamente extendida, sino la dominancia de un pequeño grupo de 5 autores que están bien posicionados en la red y que están también posicionados en las distancias más cortas entre los otros autores. Este indicador muestra aquellos autores que son intermediarios entre otros dos del mismo grupo. Existe también un grupo de 89 autores con grado de intermediación igual a cero

Tabla 3: Grado de intermediación

\begin{tabular}{lcc} 
Autores & Betweenness & nBetweenness \\
\hline------------------------ & 1.369 \\
Russell-Barnard-JM & 143.833 & 0.581 \\
Rio-JA-del & 61.000 & 0.357 \\
Narváez-Berthelemot-N & 37.500 & 0.228 \\
Michan-Aguirre-L & 24.000 & 0.181 \\
Ramírez-Godoy-ME & 19.000 & 0.133 \\
Macías-Chapula-CA & 14.000 & 0.105 \\
Licea-de-Arenas-J & 11.000 & 0.086 \\
Cortes-HD & 9.000 & 0.076 \\
Gorbea-Portal-S & 8.000 & 0.057 \\
Kostoff-RN & 6.000 & 0.048 \\
Ramírez-Romero-AM & 5.000 & 0.029 \\
García-EO & 3.000 & 0.019 \\
Luna-Morales-ME & 2.000 & 0.008 \\
Galina-Russell-I & 0.833 & 0.008 \\
Galina-CS & 0.833 &
\end{tabular}

Fuente: Datos de los autores.

Para medir la influencia de los autores, se eligieron todos los nodos a excepción de unos cuantos que mostraron grado cero y se contó la frecuencia de los grados. Con estos datos se trazó la distribución de la probabilidad de la densidad de probabilidad como se muestra en la Figura 3.

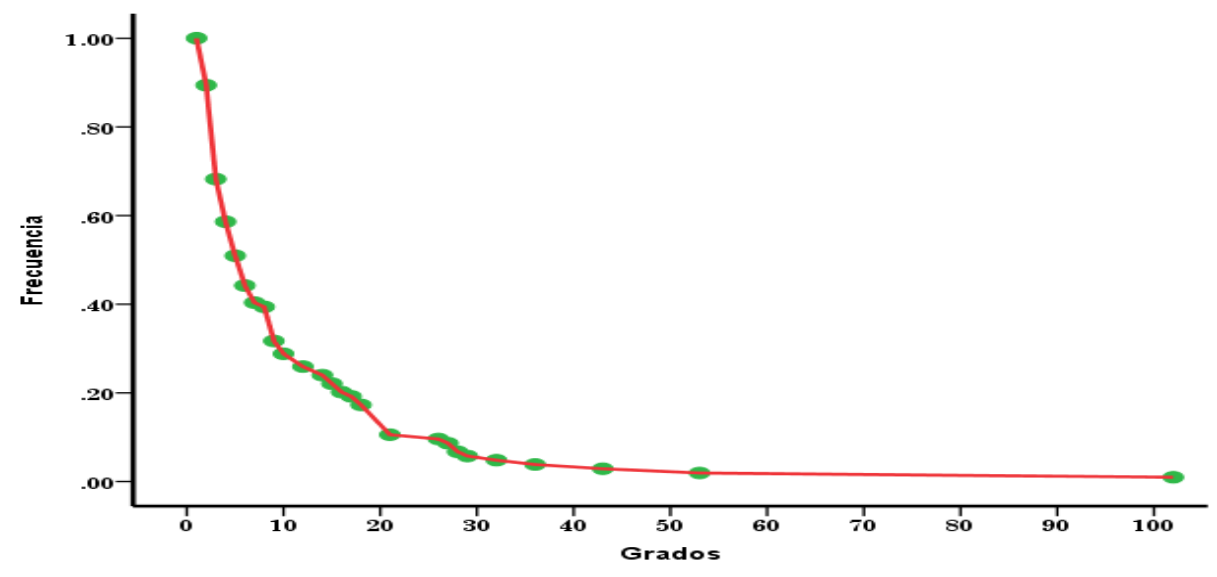

Figura 3: Probabilidad de la densidad de los autores de la bibliometría mexicana Fuente: Datos de los autores.

La frecuencia de los grados se aproximan a la distribución de Poisson; esto quiere decir que la mayoría de los autores solo trabajan en colaboración con pocos 
otros autores. Pero como hay varios autores que publican en coautoría con numerosos autores, es esta pequeña parte que se convierten en los nodos centrales de la red y desempeña un papel vital en la red de la bibliometría mexicana.

La Tabla 4 muestra la distribución estadística de los grados de intermediación de la red de la BM. Los valores máximo y mínimo varían de 144 a cero, con un coeficiente de variación igual a 251; esto significa que muchos autores no tienen ninguna conexión con los otros miembros de la red y solo reciben información pero no proporcionan información. La media del grado de intermediación es igual a 3.327 lo que es bajo, ya que indica que en media cada autor solo tiene tres relaciones con otros autores. El coeficiente de variación es igual a 4.765. A pesar de observarse una gran variación en los valores del grado de intermediación el índice de centralización general de la red es muy bajo $1.35 \%$ indicando que existe una centralización de los autores en la red, en este caso, alrededor de Russell-Barnard-JM.

Tabla 4: Distribución estadística de los grados de intermediación

\begin{tabular}{llll} 
& & Betweenness & nBetweenness \\
\hdashline 1 & Mean & 3.327 & 0.032 \\
2 & Std Dev & 15.854 & 0.151 \\
3 & Sum & 346.000 & 3.293 \\
4 & Variance & 251.342 & 0.023 \\
5 & SSQ & 27290.666 & 2.473 \\
6 & MCSSQ & 26139.551 & 2.368 \\
7 & Euc Norm & 165.199 & 1.572 \\
8 & Minimum & 0.000 & 0.000 \\
9 & Maximum & 143.833 & 1.369 \\
10 & N of Obs & 104.000 & 104.000
\end{tabular}

Fuente: Datos de los autores.

Network Centralization Index $=1.35 \%$

\subsection{Grado de cercanía}

Para conocer la capacidad que tiene un autor de colaborar con los demás autores se calcula el grado de cercanía (InCloseness). Esta medida se puede definir como la capacidad que en promedio tiene un autor de estar más cerca a los otros autores en la red. Calcula la distancia existente entre un autor hasta los otros autores de la red. Los mayores valores obtenidos sugieren que existe una mayor capacidad de colaboración dentro de la red. El grado de cercanía normalizado (Incloseness) es un valor estandarizado que varía de 1 a 100 y donde el valor más alto indica que el autor está más cerca a los otros autores colaboradores. Autores con bajos valores de cercanía pueden ser vistos como aquellos que van a tener más dificultades de acceder a la información dentro de la red. Responde a la pregunta cuán rápidamente un autor puede conectarse con los demás autores de la red. Un autor con alto grado de cercanía generalmente tiene un rápido acceso a otros autores en la red; es el camino más corto para llegar a otros autores; está mucho más cercano a los otros autores; tiene alta visibilidad sobre lo que está sucediendo en la red. Cuanto más corta en promedio es la distancia de un autor a los otros autores en la red, mayor será la cercanía, es decir, el autor está más cerca/próximo de muchos otros autores de la red.

La Tabla 5 muestra los valores de las medidas de cercanía estimados para autores de la red sobre la BM con valores mayores a 1.000. InCloseness indica la 
distancia existente desde los otros autores hasta el autor más central; OutCloseness indica la proximidad de un autor con los otros autores en la red. El autor Sierra-Flores$\mathrm{MM}$ es el que posee mayor inCloseness (1.651), pero bajo outCloseness (0.962), lo que indica que puede ser rápidamente alcanzable por otros pares de la red, pero no puede alcanzar rápidamente a los otros autores de la red. El autor Sánchez-Pereyra-A es el siguiente más cercano. Luego vienen los autores Waast-R y Zarco-L. En el caso de la autora Russell-Barnard-JM muestra un inCloseness de 1.558 más bajo en relación a los anteriores autores pero al mismo tiempo muestra un outCloseness mayor que los demás (1.000), esto indica que a pesar de ser poco difícil acceder a ella, sin embargo ella está más acerca a los otros autores que los demás. Lo mismo puede afirmarse con respecto a Narváez-Berthelemot- $\mathrm{N}$ (inCloseness $=1.031$, outCloseness $=1.074$ ), Ramírez-Romero-AM (inCloseness $=1.010$, outCloseness $=1.063$ ) y otros. Sin embargo, si se redondeasen estos números a la cifra más próxima apenas los primeros cinco autores mostrarían un inCloseness igual a 2.000 y un outCloseness también igual a 1.000, indicando que esta es una red donde todos pueden ser alcanzados fácilmente y están bastantes cercanos unos a los otros. Es una red de fácil circulación y comunicación.

Tabla 5: Grados de cercanía

\begin{tabular}{lcc} 
Autores & inCloseness & outCloseness \\
\hline Sierra-Flores-MM & 1.651 & 0.962 \\
Sánchez-Pereyra-A & 1.575 & 0.962 \\
Waast-R & 1.575 & 0.962 \\
Zarco-L & 1.574 & 0.962 \\
Russell-Barnard-JM & 1.558 & 1.000 \\
Wagner-C & 1.174 & 0.962 \\
Tshiteya-R & 1.161 & 0.971 \\
Smith-C & 1.147 & 0.980 \\
Smith-A & 1.134 & 0.990 \\
Rio-JA-del & 1.123 & 1.052 \\
Rodea-Castro-ID & 1.052 & 0.962 \\
Narváez-Berthelemot-N & 1.031 & 1.074 \\
Valles-Valenzuela-J & 1.020 & 0.962 \\
Malpohl-G & 1.020 & 1.063 \\
Ramirez-Godoy-ME & 1.010 & 1.010 \\
Romero-RD & 1.010 & 0.962 \\
Leydesdorff-L & 1.010 & 1.074 \\
Santillan-Rivero-EG & 1.010 & 0.971 \\
Mesa-ME & 1.010 & 0.962 \\
Ramírez-Romero-AM & 1.010 & 1.063 \\
Morrone-JJ & 1.010 & 0.962 \\
Zúñiga-Roca-MF & 1.000 & 0.962 \\
Licea-de-Arenas-J & 1.000 & 1.000 \\
Michan-Aguirre-L & 1.000 & 1.020 \\
Porras-A & 1.000 & 1.010 \\
Sotolongo-Aguilar-G & 1.000 & 0.962 \\
Kostoff-RN & 1.000 & 1.098
\end{tabular}

Fuente: Datos de los autores.

La Tabla 6 muestra la distribución estadística de los grados de cercanía de la red de la BM. La media es similar tanto para inCloseness (1.015) como para outCloseness (1.005), esto significa que la distancia media de un autor con otros autores es casi la 
misma que la distancia de los otros autores a los autores relevantes de la red. La desviación estándar y la media indican si la población estudiada es homogénea o heterogénea en relación con su variabilidad. De ambos valores se estiman los coeficientes de variabilidad de la red (dividiendo la desviación estándar entre la media y multiplicando el resultado por 100) tanto para inCloseness como para outCloseness. Los coeficientes de variación respectivos son de $13.3 \%$ para inCloseness y de $5.2 \%$ para outCloseness, es decir, que es más fácil que las información llegue a los autores centrales que esta información salga de los autores centrales hacia los demás autores integrantes de la red de bibliometría mexicana.

Tabla 6: Distribución estadística de los grados de cercanía

\begin{tabular}{llcc} 
& & inCloseness & outCloseness \\
\hline 1 & Minimum & 0.962 & 0.962 \\
2 & Average & 1.015 & 1.005 \\
3 & Maximum & 1.651 & 1.217 \\
4 & Sum & 105.533 & 104.532 \\
5 & Standard Deviation & 0.135 & 0.052 \\
6 & Variance & 0.018 & 0.003 \\
7 & SSQ & 108.985 & 105.353 \\
8 & MCSSQ & 1.897 & 0.287 \\
9 & Euclidean Norm & 10.440 & 10.264 \\
10 & Observations & 104 & 104
\end{tabular}

Fuente: Datos de los autores.

\subsection{Distancia geodésica (Eigenvector)}

La distancia geodésica indica el esfuerzo que hace un autor para alcanzar a otro autor en la red. Mide la distancia media de los autores para llegar a todos los demás autores. Distancias más cortas significan informaciones más rápidas, más seguras, y más precisas en la transmisión de informaciones a compartir. Se expresa como el promedio del número de pasos para llegar a todos los participantes de la red. Los valores más bajos indican grupos o individuos en mejores condiciones de aprovechar el conocimiento. Para la red de coautorías de la BM se encontró que la distancia media más corta que hay entre los autores de la red es de 1.074 autores, por lo tanto los autores de la BM aprovechan muy bien el flujo de comunicaciones en la red de coautorías.

Es una forma de identificar a los autores más centrales, aquellos con menos lejanía que los otros. Es una medida de la popularidad o centralidad de los autores conocidos. No mide si se tienen muchos contactos, sino si se tienen buenos contactos. Mide el grado en que un autor está conectado con otros autores que están bien conectados. Permite identificar a los autores colocados más centralmente en la estructura de la red. Valores más altos indican mayor centralidad de los autores en la red. El autor que tiene la puntuación más alta es el que está conectado a muchos autores que también están bien conectados. El "eigenvector" de las distancias geodésicas es una medida de centralidad dirigida a encontrar a los actores centrales de una estructura global o general, prestando menos atención a los patrones locales. Permite medir la importancia de un autor en la red.

La Tabla 7 muestra los valores encontrados de las distancias geodésicas de la red de coautorías de la BM. Solamente se presentan los autores que obtuvieron valores cercanos a 1 (uno). La autora Russell-Barnard-JM es la mejor conectada y está conectada a otros autores también muy bien posicionados en la red. Luego viene la 
autora Narvaez-Berthelemot-N. También los autores Collazo-Reyes-F, Rio-JA-del y Luna-Morales-ME pueden ser considerados como bien conectados. En general estos 16 autores listados en la Tabla 7 pueden ser considerados como bien conectados y que están también conectados a otros autores muy bien posicionados en la red de la bibliometría mexicana. El resto de los autores son más bien periféricos y poco conectados.

Tabla 7: distancias geodésicas de la red de coautores

\begin{tabular}{lcc} 
Autores & Eigenvec & nEigenvec \\
\cline { 1 - 2 } Russell-Barnard-JM & 0.570 & 80.610 \\
Narváez-Berthelemot-N & 0.343 & 48.567 \\
Collazo-Reyes-F & 0.302 & 42.664 \\
Rio-JA-del & 0.294 & 41.581 \\
Luna-Morales-ME & 0.289 & 40.836 \\
Cortes-HD & 0.246 & 34.754 \\
Ainsworth-S & 0.213 & 30.089 \\
Pérez-Angón-MA & 0.187 & 26.385 \\
Kostoff-RN & 0.125 & 17.731 \\
Galina-Russell-I & 0.116 & 16.420 \\
Arvanitis-R & 0.112 & 15.802 \\
Gaillard-J & 0.100 & 14.135 \\
Karypis-G & 0.092 & 12.990 \\
García-EO & 0.091 & 12.913 \\
Rosas-AM & 0.082 & 11.563
\end{tabular}

Fuente: Datos de los autores.

La Tabla 8 muestra la distribución estadística de la distancia geodésica (Eigenvector) de la red de la BM. La media es 0.044 para el eigenvector y 6.191 para el eigenvector normalizado, pero la desviación estandarizada es mayor para ambos eigenvectores, esto indica que existe variabilidad en la centralidad, es decir, estos valores sugieren que existen grandes desigualdades en la centralidad o poder de los autores de la red de BM. El índice de centralización de la red respecto del eigenvector es del 83\%.

Tabla 8: Distribución estadística de la

Distancia geodésica (Eigenvector)

\begin{tabular}{llcl} 
& & Eigenvec & nEigenvec \\
\hline 1 & Mean & 0.044 & 6.191 \\
2 & Std Dev & 0.088 & 12.409 \\
3 & Sum & 4.553 & 643.825 \\
4 & Variance & 0.008 & 153.984 \\
5 & SSQ & 1.000 & 20000.002 \\
6 & MCSSQ & 0.801 & 16014.322 \\
7 & Euc Norm & 1.000 & 141.421 \\
8 & Minimum & -0.000 & -0.000 \\
9 & Maximum & 0.570 & 80.610 \\
10 & N of Obs & 104.00 & 104.00 \\
11 & N Missing & 0.000 & 0.000 \\
Network centralization index $=83.35 \%$ &
\end{tabular}

Fuente: Datos de los autores. 


\section{CONCLUSIONES}

Este artículo se propuso analizar la red de coautorías existentes en las publicaciones sobre las metrías (bibliometría, informetría, cienciometría, patentometría, etc.) en México, desde la década de los 70s hasta diciembre de 2012. Se encontraron 459 documentos producidos y publicados en ese periodo de investigación. El 37\% de esos documentos (171) fueron producidos sin colaboración; por lo tanto 288 (63\%) documentos fueron producidos en colaboración por dos $(25 \%)$, tres $(21 \%)$, cuatro (9\%), cinco (6\%), etc. autores. Solamente $17 \%$ de los documentos (77 publicaciones) fueron producidos por cuatro o más autores colaboradores. Los productores y colaboradores de estos 288 documentos fueron objeto de análisis de esta investigación.

Se identificó una red compuesta básicamente de 8 grupos diferenciados. Un grupo central con la posición dominante del autor Jane Margaret Russell Barnard que lidera 18 autores colaboradores; seguido de otro grupo liderado por la autora Judith Licea de Arenas y dos grupos menores, una liderada por el autor E. Aguado López y el otro por CA Macías Chapula. Se observa aun otro grupo formado por tres autores altamente colaboradores liderado por el autor ME Ramírez Godoy. Existen también tres grupos de dos autores colaboradores marginales y aislados del resto de los miembros de la red. Como esta red está altamente centralizada la eventual remoción del autor central podría producir una rápida fragmentación en pequeños grupos de autores aislados.

La densidad global de la red fue del 4.65\% ( \pm 0.445 ), indicando que estamos en presencia de una red con una densidad de comunicación baja que hace una utilización efectiva de una quinta parte de los vínculos reales sobre el total de vínculos posibles entre todos los autores de la red. En otras palabras, solo se están utilizando el 5\% de las relaciones posibles y se están perdiendo el 95\% de ellas. La distancia existente entre los autores de la red es de 1.074 autores, por lo tanto, los autores de la bibliometría mexicana hacen buena utilidad del flujo de comunicaciones en la red.

En relación al grado de centralidad se identificaron 23 autores como los componentes estructurales de la red de bibliometría mexicana. Estos autores poseen un mayor valor de grado de centralidad indicando que tienen el mayor número de conexiones y por lo tanto mayor sociabilidad en la red. Son los que tienen mayor capacidad de intercambiar o diseminar informaciones con los demás pares y pueden ser caracterizados como los autores más influyentes de la bibliometría mexicana. Se identificó una autora (Jane Margaret Russell Barnard) que posee el mayor grado de centralidad y juega un papel estructural en la bibliometría mexicana, ya que es la autor que más ha colaborado con otros autores, es decir, la que tiene el mayor número de conexiones; su grado de centralidad duplica en tamaño al siguiente más cercano significando que es la que recibe más solicitaciones de colaboración de parte de otros autores.

Para el grado de cercanía se encontró que un autor (MM Sierra Flores) posee mayor inCloseness (1.651), pero bajo outCloseness (0.962) esto indica que puede ser rápidamente alcanzable por otros pares de la red, pero no puede alcanzar rápidamente a los otros autores de la red. Caso similar es el de los autores A Sanchez-Pereira, R Waast, L. Zarco y Jane Margaret Russell Barnard con grado de cercanías similares. La distribución estadística de los grados de cercanía indica que tanto para inCloseness como para outCloseness la distancia media de un autor con otros autores es la misma que la distancia de los otros autores con los autores relevantes de la red. Los coeficientes de variación indican que tanto la lejanía como la cercanía son más o menos homogéneas. 
En relación con el grado de intermediación se encontró que no existe una red ampliamente extendida, sino la dominancia de una autora (Jane Margaret Russell Barnard) y luego un pequeño grupo de cinco autores. El autor con mayor grado de intermediación se colocó a casi el doble de distancia del segundo más próximo y éste a su vez casi tres veces más alejado del tercero. El resto de autores son nulos en intermediación.

En relación con la distancia geodésica se identificó una única autora (Jane Margaret bien conectado con otros autores también muy bien posicionados en la red. Luego le siguen otros seis autores medianamente bien conectados. El resto de los autores son más bien periféricos. La distribución estadística de la distancia geodésica (Eigenvector) de la red de la bibliometría mexicana confirma que existe mucha variabilidad en la centralidad, significando que existe grandes desigualdades en la centralidad o poder de los autores de la red.

\section{BIBLIOGRAFÍA}

Aguado-López, Eduardo, Rogel-Salazar, Rosario, Garduño-Oropeza, Gustavo, BecerrilGarcía, Arianna, Zúñiga-Roca, María Fernanda, Velázquez-Álvarez, Alejandro. Patrones de colaboración científica a partir de redes de coautoría. Convergencia: Revista de Ciencias Sociales, (núm., especial IA):225-228, 2009.

Barabási, A. L.; Jeong, H.; Neda. Z.; Ravasz, E.; Schubert, A. \& Vicsek, T. Evolution of the social network of scientific collaborations (2002). Physica A: Statistical Mechanics and its Applications. 311(3-4):590-614, 2002.

Balancieri, R. et al. A análise de redes de colaboração científica sob as novas tecnologias de informação e comunicação: um estudo na Plataforma Lattes. Ciência da Informação, Brasília, DF, 34(1):64-77, 2005.

Baranger, Denis. Sobre estructuras y capitales: Bourdieu, el análisis de redes, y la noción de capital social. Revista de Antropología Avá (2):41-63, 2000.

Borgatti, S.P.; Foster, P.C. The network paradigm in organizational research: a review and Typology. Journal of Management, 29(6):991-1013, 2003.

Bourdieu, Pierre. The Forms of Social Capital. En: Handbook of Theory and Research for the Sociology of Education, ed. J. Richardson, N.Y. Greenwood, 1985.

Bourdieu, Pierre \& Wacquant, Loïc. Una invitación a la sociología reflexiva. Buenos Aires, Argentina: Siglo XXI Editores, 2005.

Bufrem, Leilah Santiago; Gabriel Junior, Rene Faustino \& Sorribas, Tidra Viana. Redes sociais na pesquisa científica da área de ciência da informação. DataGramaZero - Revista de Informação, 12(3) Ago. 11, ARTIGO 01.

Castells, Manuel. Comunicación y poder. Madrid: Alianza Editorial, 2009.

Collazo-Reyes, Francisco; Luna Morales, María Elena \& Luna Morales, Evelia.

Aproximación a las formas de organización de la producción científica a través de redes de coautoría. Avance y Perspectiva, 4(3), 2012. Disponible en:

http://avanceyperspectiva.cinvestav.mx/1866/aproximacion. Consultado en 15 de abril 2015

Crane, Diana. Invisible colleges: diffusion of knowledge in scientific communities. Chicago and London: The University of Chicago Press, 1972.

Delgado López-Cózar, Emilio; Torres-Salinas, Daniel; Jiménez-Contreras, Evaristo \& Ruiz-Pérez, Rafael. Análisis bibliométrico y de redes sociales aplicado a las tesis bibliométricas defendidas en España (1976-2002): temas, escuelas científicas y redes académicas. Revista Española de Documentación Científica, 29(4):493-524, 2006. 
Diccionario de la Lengua Española. 22a. Ed. Madrid, España, Real Academia Española, 2001.

Fleck, L. The genesis and development of a scientific fact. Chicago: The Chicago University Press; 1979.

Graeml, Alexandre Reis; Macada, Marie Anne \& Rosson, Luciano. Redes sociais e intelectuais em Administração da Informação: uma análise cientométrica do período 1997-2006. Informação \& Sociedade: Estudos, 20(1):95-110, 2010.

Hanneman, R.; Riddle, M. Introduction to social network methods, 2005. Disponible en: http://faculty.ucr.edu/ hanneman/nettext/C1_Social_Network_Data.html. Consultado en: 14 de abril de 2015.

Iñiguez, L.; Muñoz, J.; Peñaranda, M. C. Y Martínez, L. M. “La psicología social en España: estructuras de comunidades". Redes, 10(3), 2006.

Katz, J. S. \& Martin, B. R. What is research collaboration? Research Policy, 26(1):1-18, 1997.

Leta Jacqueline \& Cruz, Brito Carlos Henrique de. A produção científica brasileira. In: Viotti, EB \& Macedo, MM (Editores). Indicadores de ciência, tecnologia e inovação no Brasil. Campinas: Editora da Unicamp; 2003. p. 121-168.

Nascimento, Bruna S. do. A Ciência da Informação no Brasil: um retrato da área através do estudo de autoria e da análise das redes de colaboração científica. Dissertação (Mestre em Ciência da Informação) --Universidade Federal da Bahia, Instituto de Ciência da Informação, Programa de Pós-Graduação em Ciência da Informação. Salvador, Bahía, 2011.

Newman, M. E. J. Who is the best connected scientist?: a study of scientific coauthorship networks. Santa Fé: The Santa Fé Institute, 2000. Paper 00-12-064. Price, John Derek de Solla \& Beaver, Donald de B. Collaboration in an invisible college. American Psychologyst, 21:1011-1018, 1966.

Price, John Derek de Solla. Little science, big science. New Cork: Columbia University Press, 1963.

Parreiras, Fernando Silva; Silva, Antônio Braz de Oliveira; Matheus, Renato Fabiano \& Brandão, Wladmir Cardoso. RedeCI: colaboração e produccion científica em ciência da informação no Brasil. Perspect. ciênc. inf., Belo Horizonte, 11(3):302-317, 2006. Pinto, Adilson Luiz; Efrain-García, Preiddy; Rodríguez Barquín, Beatriz Ainhize \& Moreiro González, José Antonio. Indicadores científicos na literatura em bibliometria e cientometria através das redes sociais. Brazilian Journal of Information Science, 1(1):58-76, 2007.

Sadoughi, Farahnaz; Valinejadi, Ali; Serati Shirazi, Mansoureh \& Khademi, Rouhallah. Social Network Analysis of Iranian Researchers on Medical Parasitology: A 41 Year CoAuthorship Survey. Iran J Parasitol, Vol. 11, No. 2, Apr -Jun 2016, pp. 204-212.

Urbizagástegui Alvarado, Rubén \& Restrepo Arango, Cristina. Acercamiento a los estudios bibliométricos, cienciométricos e informétricos en México. En: XV ENANCIB: Alem das Nuvens: expandindo as fronteiras da Ciência da Informação, Belo Horizonte, Minas Gerais,Brasil, de 27 a 31 de outubro de 2014.

Silva, Antônio Braz de Oliveira; Parreiras, Fernando Silva; Matheus, Renato Fabiano \& Brandão, Wladmir Cardoso. Redes de co-autoria dos professores da ciência da informação: um retrato da colaboração científica dessa disciplina no Brasil. Vargas-Quesada, Benjamín; Minguillo, David; Chinchilla-Rodríguez, Zaida \& MoyaAnegón, Félix de. Estructura de la colaboración científica española en Biblioteconomía y Documentación (Scopus 1999-2007). Revista Interamericana de Bibliotecología, 33(1):105-123, 2010. 
Wasserman, Stanley \& Faust, Katherine. Social network Analysis. Methods and Applications. Cambridge University Press: Cambridge, 1994.

Yan, Erjia \& Ding, Ying. Applying centrality measures to impact analysis: A coauthorship network analysis. Journal of the American Society for Information Science and Technology, 60(10):2107-2118, 2009.

Ziman, John M. Conhecimento público. Belo Horizonte: Ed. Itatiaia, 1979.

Zoss, Angela. Seeding a Field: The Growth of Bibliometrics through Co-authorship Ties. Bulletin of the ASIST, 38(6):39-44, 2012

Zuccala, A. Modeling the Invisible College. Journal of the American Society for Information Science and Technology, 57:152-168, 2006.

ANEXO A: Términos de busca

México / Índice h / Elitismo / Frente de investigación / Regla 80/20 / Obsolescencia de la literatura / Crecimiento de la literatura / Vida media / Teoría epidémica / Visibilidad / Índice de Pratt / Índice de Price / Ley de Price / Indicadores bibliométricos / Ley de Goffman / Ley de Bradford / Ley de Lotka / Ley de Zipf / Punto de transición / Colegios invisibles / Factor de impacto / Factor de inmediatez / Análisis de citas / Acoplamiento bibliográfico / Cocitación / Redes sociales / Co-autoría / Colaboración científica / Índice de colaboración / Coeficiente de colaboración / Circulación de la colección / Núcleo básico de periódicos / Indicadores en ciencia y tecnología / Bibliometría / Cienciometría / Informetría / Patentometría / Archivometría / Bio-bibliometría / Librometría / Tecnometría / Webmetría

ANEXO B: Bases de datos consultadas

INFOBILA de México / Humanindex / Catálogo de la biblioteca del IIBI/UNAM / Periodica / Redalyc / Scielo México / Scielo Brasil / Scielo Venezuela / Scielo Colombia / Scielo Chile / Scielo Argentina / Scielo Bolivia / Library Literature \& Information Science Full Text / Library and Information Science Abstract (LISA) / Library, Information Science \& Technology Abstracts (LISTA) / WorldCat / HAPI / ArticleFirst / Science Citation Expanded Index / Web of Science / Scopus / JSTOR / Google / Google Scholar / ISOC / ICYT / Dialnet / Agrícola / Biosis / CAB Abstracts / Medline / y otras 120 bases de datos existentes en la Universidad de California en Riverside, USA. 